Meta

Journal des traducteurs

Translators' Journal

\title{
Psychanalyse et traduction
}

\section{François Peraldi}

Volume 27, numéro 1, mars 1982

Psychanalyse et traduction

URI : https://id.erudit.org/iderudit/003817ar

DOI : https://doi.org/10.7202/003817ar

Aller au sommaire du numéro

Éditeur(s)

Les Presses de l'Université de Montréal

ISSN

0026-0452 (imprimé)

1492-1421 (numérique)

Découvrir la revue

Citer cet article

Peraldi, F. (1982). Psychanalyse et traduction. Meta, 27(1), 9-25.

https://doi.org/10.7202/003817ar

Ce document est protégé par la loi sur le droit d'auteur. L'utilisation des services d'Érudit (y compris la reproduction) est assujettie à sa politique d'utilisation que vous pouvez consulter en ligne.

https://apropos.erudit.org/fr/usagers/politique-dutilisation/
Cet article est diffusé et préservé par Érudit.

Érudit est un consortium interuniversitaire sans but lucratif composé de l’Université de Montréal, l'Université Laval et l'Université du Québec à Montréal. Il a pour mission la promotion et la valorisation de la recherche. https://www.erudit.org/fr/ 


\title{
PSYCHANALYSE ET TRADUCTION
}

\author{
François Peraldi
}

Il paraîtra sans doute tout à fait surprenant à un bon nombre de traducteurs qu'on puisse vouloir conjoindre la psychanalyse et la traduction et, $a$ fortiori, qu'on ait consacré un numéro spécial de la revue Meta à une telle conjonction. Je parle de ces traducteurs pour qui la traduction n'est qu'une simple opération technique qui consiste à faire passer le contenu d'un message d'une langue de départ 1 dans une langue d'arrivée 2 . En termes un peu plus linguistiques, disons à faire passer le(s) même(s) signifié(s) - fussent-ils agrémentés de leur 'valeur' dégagée en référence non seulement au contexte, mais à ce que $M$. Pergnier ${ }^{1}$, par exemple, nomme les "paramètres situationnels du message» - d'une structure signifiante A dans une structure signifiante $B$, en réduisant les distorsions, trahisons et pertes sémantiques au maximum.

Mais il existe également un bon nombre de psychanalystes qui ne seront pas moins éberlués de ce rapprochement qui leur paraîtra, à tout le moins, incongru. Je parle de ceux pour qui la psychanalyse n'est qu'une technique thérapeutique, une cousine un peu bizarre, et plus ou moins ouvertement décriée, de ce qui s'affiche aujourd'hui dans le champ quelque peu hétéroclite de la «psychiatrie dynamique» qui, ne se contentant plus d'être médicale, ce en quoi elle a toujours échoué, se veut désormais «bio-psycho-sociale» ${ }^{2}$. Pour eux la psychanalyse se définit, mal d'ailleurs, par sa méthode: une certaine écoute, son objet: la maladie mentale ou du moins certains de ses aspects les moins dévastateurs, et par son but : faire passer un patient d'un système de comportements et de représentations défini comme pathologique à un système de comportements et de représentations différent du premier et plus ou moins proche d'un modèle défini comme «norme». Le passage du système 1 au système 2 devant permettre l'élimination d'un ensemble de symptômes centrés par la «souffrance» du sujet et définis par leurs caractères a- ou anti-sociaux. Le «transfert psychanalytique» est le moteur de la transformation de ces patients névropathes ou psychopathes en un individu comme tout le monde, ce que Jacques Schotte nomme, non sans quelque ironie: un normopathe ${ }^{3}$.

Mis à part le passage d'un système à un autre avec quelque chose qui se perd irrémédiablement (le signifiant en traduction, les symptômes en psychana-

1. Maurice Pergnier (1981): Théorie de la linguistique et théorie de la traduction, Meta, vol 26, $\mathrm{n}^{\circ}$ 3, Presses de l'Université de Montréal.

2. C'est, par exemple, ce qui se dégage d'un volumineux Précis de pratique psychiatrique, publié chez Stanké, 1982.

3. Jacques Schotte a proposé cette notion au cours d'une conférence présentée à l'Université de Montréal dans le cadre de notre séminaire en criminologie, en février 1980. 
lyse) pour que quelque chose soit préservé coûte que coûte (le signifié + le sens en traduction, le noyau sain ou la structure normopathique du sujet en psychanalyse), on voit mal, a priori, ce qui peut rapprocher ces deux pratiques qui, d'un point de vue purement empirique, semblent poursuivre des fins radicalement différentes dans deux domaines apparemment tout à fait hétérogènes.

Tous les traducteurs ne sont toutefois pas encore réductibles à leurs outils ni aux méthodes qui en guident l'utilisation, même si dans certains lieux de formation des dits traducteurs on peut entendre parfois prôner un enseignement qui serait fondé sur la «passivation des étudiants" (sic), c'est-à-dire leur assujettissement absolu aux exigences méthodologiques de leur pratique : ce qui définit le rapport du sujet à une idéologie pratique plutôt qu'à une science.

De leur côté, tous les psychanalystes ne sont pas ces shrinks assujettis à un corpus technique rigoureux (comme celui complaisamment décrit par Greenson dans son Technique and Practice of Psychoanalysis) ${ }^{4}$, et dont l'ampleur de l'assujettissement permet aux institutions dont ils dépendent de les reconnaître ou non comme psychanalystes et leur pratique comme relevant, ou pas, de la psychanalyse qui devient de ce fait une sorte d'Idée inaccessible au sens platonicien du terme.

Il est des traducteurs et des psychanalystes qui, entre, d'une part, la pratique qui constitue leur activité quotidienne et la technique sur laquelle elle prend appui et, d'autre part, leur position de sujet de la science psychanalytique ou de la traduction, introduisent cette activité réflexive qui semble toujours être très décriée en Amérique du Nord: la théorisation. Il est des traducteurs et des psychanalystes qui font, qui feront toujours de cette réflexion théorique non pas une sorte de construction gratuite et superflue (intellectualisante dit-on non sans quelque mépris) réservée aux seuls pontes institutionnels pour se représenter ce qu'ils font en termes plus ou moins sophistiqués, mais au contraire, le fondement même de leur pratique et des techniques qu'ils sont amenés à ré-évaluer sans cesse, afin, précisément, d'échapper au terrorisme trompeur des faits et des phénomènes fournis par l'expérience immédiate, et afin de se soustraire à un empirisme naïf que le «Nouvel esprit scientifique» a finalement reconnu comme l'un des obstacles les plus redoutables qu'il ait à surmonter dans le champ des sciences du vingtième siècle ${ }^{5}$.

C'est cette activité de théorisation qui constitue le repère le plus sûr de leur pratique, c'est elle qui donne à leurs méthodes et à leurs techniques leur sens véritable, c'est elle, et non la position de pouvoir toujours arbitraire de tel superviseur en traduction ou de tel didacticien en psychanalyse dont le savoir-supposé se résume le plus souvent à un argument de force. L'activité théorique est la subversion la plus efficace de ces hiérarchies de pouvoir fondé sur le savoir-supposé, elle exclut la médiation autoritariste d'un tiers pour devenir elle-même l'activité médiatrice et réflexive entre un sujet (à entendre ici

4. Ralph Greenson (1975): The Technique and Practice of Psychoanalysis, I.U.P.

5. Les conceptions épistémologiques avancées ici sont celles exposées par Gaston Bachelard (1967): la Formation de l'esprit scientifique, Vrin, et (1934): le Nouvel esprit scientifique P.U.F. 
comme sujet de la science) et sa pratique puisque, en tant que discours théorique, elle comporte et propose nécessairement les exigences logiques et épistémologiques propres à la science en question.

Il s'avérera d'ailleurs toujours que la théorisation est un maître infiniment plus exigeant que tous les maîtres, qu'ils soient superviseurs ou didacticiens et, assurément, infiniment plus juste puisqu'au niveau de l'exigence théorique aucun pouvoir n'est plus en jeu, pour le praticien, autre que la maitrise conceptuelle de sa propre pratique; mais une maîtrise bien particulière en ceci : qu'elle est continuellement remise en jeu, en question, dans la tension dialectique qui s'instaure entre la pratique et l'effort de conceptualisation de cette pratique que nous nommons théorisation (plutôt que théorie). La théorisation est, à proprement parler, un «effort de pensée», dans le sens le plus élevé, le plus rigoureux et le plus exigeant qu'il convient, avec Heidegger ${ }^{6}$ par exemple et contre une certaine compréhension linguistico-psychologisante du terme assez bien illustrée par un auteur comme Jerry Fodor ${ }^{7}$, de donner au mot «pensée».

C'est ici, au niveau de la théorisation, que la question du rapprochement de la psychanalyse et de la traduction se présente sous un jour très différent de l'obscurantisme, quelque peu caricatural, sous l'angle duquel je l'ai introduite. En effet, ce dont les traducteurs et les psychanalystes doivent produire la théorie, s'ils veulent faire l'effort de penser leur pratique, est une seule et même chose : le langage du sujet humain. Je parle du langage non pas seulement en tant que comportement (à quoi le réduisent les behavioristes de tous bords) ni en tant qu'instrument de communication (à quoi le réduisent les théoriciens de la communication et cybernéticiens de tous genres), ni en tant que système syntagmatico-paradigmatique, plus ou moins génératif et/ou transformationnel (à quoi le réduisent les linguistes saussuriens, néo-saussuriens et chomskiens...); mais en tant qu'il est la condition primordiale de tout rapport possible dans le monde habité par l'homme lorsque celui-ci est considéré comme sujet, c'est-à-dire en tant qu'il parle et que, de ce fait, il est parlé.

Car la parole, comme fonction du langage, n'est pas seulement un médium, encore moins un instrument, à la disposition de sujets qui lui pré-existeraient et se l'échangeraient comme les enfants leurs billes d'agathe contre un petit bateau à voiles. La psychanalyse a montré que la parole, comme fonction du langage, a, sur le petit d'homme, des effets considérables puisqu'en somme elle le fait passer de sa situation proprement animalière au statut de sujet social dont toute la sociabilité - son rapport aux autres sujets autant qu'à lui-même et au monde objectal - est dominée, conditionnée, surdéterminée par la fonction de la parole dans le champ du langage. Le psychanalyste, à chaque moment de sa pratique, ne cesse de vérifier que le langage n'est pas un produit du sujet humain mais, qu'au contraire, c'est le sujet humain qui est un produit, un effet du langage et de la parole. Il le vérifie et le démontre à chaque fois que, par les seuls effets de la parole, il constate que s'opèrent

6. Martin Heidegger (1967): Qu'appelle-t-on penser?, P.U.F.

7. Jerry Fodor (1975): The Language of Thought, Harvard University Press. 
des remaniements subjectifs qui peuvent se répercuter jusqu'au plus profond des structures biologiques mêmes du corps.

C'est d'un effet de ce genre qu'est née la psychanalyse et pourquoi ne pas l'évoquer ici puisqu'il nous semble particulièrement parlant?

Anna $O$. souffrait d'une hydrophobie excessivement grave entre autres symptômes qui, pour ne pas avoir d'origine organique repérable, n'en étaient pas moins bien réels : «paraphasie, strabisme convergent, troubles graves de la vue, contracture parésique totale dans le membre supérieur droit et les deux membres inférieurs, et partielle dans le membre supérieur gauche, parésie des muscles du cou... plus tard s'ajoutèrent somnambulisme et cette hydrophobie qui devait amener Anna, pour calmer sa soif, à ne manger que des fruits, des melons, etc. ${ }^{8}$. Ce n'est pas à Freud qu'il devait appartenir de découvrir les effets curatifs de la parole, mais à Anna $O$. et à son médecin traitant d'alors, le Dr Joseph Breuer. Il devait appartenir à Freud, par contre, en s'appuyant sur cette découverte, de construire la psychanalyse. On aura sans doute reconnu dans les maux dont souffrait Anna $O$., une hystérie, la grande névrose de l'époque, que l'on soignait en faisant appel à l'hypnose. L'hypnose permettait aux patients, le plus souvent des femmes, de se remémorer des événements oubliés en rapport avec leur maladie et d'une grande importance pour le médecin. Sous hypnose, Anna $O$. devait se souvenir un jour, alors que son hydrophobie avait pris une importance considérable, de «sa dame de compagnie anglaise [lorsqu'elle était enfant] qu'elle n'aimait pas et elle raconta avec tous les signes du dégoût, qu'étant entrée dans la chambre de cette personne, elle la vit faisant boire son petit chien, une sale bête, dans un verre. Par politesse, Anna n'avait rien dit. Après $m$ 'avoir énergiquement exprimé sa colère rentrée [c'est Breuer qui parle], elle demanda à boire et avala sans peine une grande quantité d'eau et sortit de son état hypnotique le verre aux lèvres; après quoi le symptôme ne se manifesta jamais plus».

Cet exemple est important en ceci :

1) qu'il montre qu'à ses débuts, le travail de l'analyse apparaît comme ce que Roman Jakobson nomme une «traduction intersémiotique " ${ }^{9}$, c'est-àdire: "l'interprétation de signes linguistiques (le récit de la scène du petit chien de la gouvernante et du dégoût d'Anna) au moyen de systèmes de signes non linguistiques (ici, corporels : l'hydrophobie, le dégoût de tout liquide entraînant l'impossibilité de boire)». La maladie, pour schématiser les choses, opère la traduction du souvenir verbal en un signe corporel complexe équivalent (l'élément commun est le dégoût de boire); le travail de l'analyse apparaît alors comme une traduction en sens inverse, une retraduction, le retour aux signes verbaux du souvenir.

2) Cet exemple nous montre également toute l'importance des systèmes sémiologiques et, en particulier, des signes verbaux en tant qu'ils constituent la trame vivante, la substance même des rapports inter-subjectifs qui fondent

8. Joseph Breuer et Sigmund Freud : Études sur l'hystérie, P.U.F.

9. Roman Jakobson (1963): «Aspects linguistiques de la traduction», dans Essais de linguistique générale, Editions de Minuit. 
le social mais en tant qu'il (le système verbal) donne à tous les systèmes sémiologiques non verbaux leur structure fondamentale. On mesurera mieux sans doute, de ce point de vue, l'immense responsabilité de quiconque ose manipuler cette trame vivante, qu'il s'agisse du psychanalyste, tout autant que du traducteur pour autant que nous situions sa pratique dans le cadre élargi de la définition tripartite que nous en a donné Jakobson.

3) Il montre enfin que les processus langagiers (en y incluant les diverses formes de traduction) ne sont pas de simples échanges d'informations dans la transparence de la conscience, mais qu'ils mettent en jeu toutes sortes de phénomènes de déplacement, de refoulement, de résistance (il a fallu qu'Anna soit sous hypnose pour qu'elle puisse se remémorer le souvenir refoulé) qui s'opposent continuellement et pour chacun d'entre nous, à la libre circulation d'une parole vraie et dont la signification serait également partagée entre tous les sujets. D'une certaine manière, nous ne pouvons pas ne pas parler, mais toute parole, de par la complexité des structures subjectives qu'elle met en place, prête nécessairement au malentendu, à la méprise.

L'ensemble de ces phénomènes subsumés par la notion double de refoulement/résistance a été minutieusement décrit par Freud. Ils sont constitutifs du clivage irrémédiable du sujet entre l'inconscient du côté duquel nous situerons le sujet, comme sujet de l'inconscient, et le moi. Un clivage (Spaltung) qui ne cesse de se manifester dans un usage paradoxal de la parole dont il faudra bien qu'un jour les théoriciens du langage prennent toute la mesure.

Freud devait reprendre les choses là où Breuer les avait laissé tomber avec quelque précipitation. En effet, le soin et l'acharnement avec lesquels cet excellent homme avait aidé Anna $O$. à déchiffrer et à retraduire ses maux en mots, au cours de ce qu'elle devait elle-même appeler non sans un certain humour sa talking cure ou encore le chimney sweeping, devait faire naître chez cette patiente jeune, belle et intelligente une reconnaissance et une affection envers un médecin aussi dévoué, bien proches de l'amour. Breuer en fut péniblement troublé; la plus grande partie des symptômes d'Anna ayant disparu, il put interrompre assez précipitamment le traitement et, si l'on en croit l'anecdote, s'enfuir avec son épouse légitime vers Venise pour une seconde lune de miel.

Freud s'était déjà aperçu en écrivant sa monographie sur les aphasies, en 1891, quatre ans avant que ne commence la psychanalyse, que les théories des localisations cérébrales de la parole, développées, après Broca par Wernicke, Lichtheim et Meynert (qui fut le maître de Freud en neurologie), ne permettaient pas une étude exhaustive des troubles de l'aphasie qui semblent pourtant plus neurologiques que psychiques. Il posa qu'il fallait bien plutôt partir des structures mêmes du langage et de la parole et de leurs diverses altérations afin d'expliquer les deux grandes formes de l'aphasie déjà dégagées. Cette hypothèse, nous sommes, rappelons-le, en 1891, devait l'amener à anticiper sur les travaux de Saussure et de Jakobson. C'est ainsi, pour être bref mais ne pas éviter un détour nécessaire tant la confusion répandue sur ces questions par les psychanalystes eux-mêmes est massive, que Freud devait observer que lorsqu'on prête attention aux énoncés de quelqu'un, on peut être attentif soit à l'image 
sonore, l'image matérielle, comme lorsqu'on corrige les épreuves d'un texte pour sa publication, soit au contenu, aux idées, aux représentations, comme lorsqu'on lit un roman passionnant dans un train sans s'occuper des fautes de typographie. Mais, fait-il remarquer, autant le contenu (ce que Saussure nommera le signifié) est flou, mouvant, insaisissable et finalement difficile à étudier, autant l'image matérielle des mots (ce que Saussure devait nommer le signifiant) est rigoureusement structurée et parfaitement analysable dans sa matérialité même. C'est donc, conclut Freud, de l'image matérielle - ce qu'il appellera plus tard le représentant de la représentation - du signifiant, pourrions-nous dire, qu'il convient de partir pour analyser les diverses formes de l'aphasie, ce qui, parce qu'il s'appuie sur des bases aussi sûres, l'amène à distinguer, bien avant Goldstein et Jakobson, les perturbations qui se manifestent au niveau de l'enchaînement des images matérielles (troubles de la contiguïté selon Jakobson) de celles qui se manifestent au niveau de la substitution de ces images matérielles (les troubles de la similarité chez Jakobson) ${ }^{10}$.

C'est précisément cette intuition-ci, maintes fois reprise, sous maintes formes différentes qui devait marquer le commencement de la psychanalyse; à savoir la nécessité théorique et méthodologique d'aborder l'étude des phénomènes que l'on regroupe sous le nom de psychiques par le biais du signifiant. Car même si Freud n'emploie jamais ce mot (qu'il appartiendra à Saussure d'imposer), c'est toujours de cela qu'il parle, ce que Lacan a démontré à plusieurs générations d'analystes y compris ceux qui feignent de l'ignorer, et sur la structure de quoi il s'appuie lorsqu'il parle d'«idées», de «représentations» et de «traces mnésiques », qu'elles soient refoulées ou non, mais compte tenu du fait que ce qui l'intéresse avant tout est ce qui est refoulé : l'inconscient.

Lorsqu'après Breuer, Freud aborde, seul, l'étude des névroses, et surtout en ces commencements lointains, de l'hystérie, puis ensuite des phénomènes psychiques plus généraux dont les névroses ne sont, en somme, que des variantes, il devait partir de l'hypothèse de Breuer que "l'hystérique souffre de réminiscences ». C'est-à-dire que, comme nous l'avons vu pour Anna O., les symptômes dont les hystériques souffrent sont la traduction dans une sémiotique corporelle, de souvenirs refoulés, de récits d'événements (réels ou fantasmatiques) chassés du conscient mais relevant d'une sémiotique verbale. Le travail d'analyse inauguré par Breuer et Anna O. étant, nous l'avons vu, une retraduction des signes corporels en signes verbaux. Mais nous avons vu également que, pour Freud, ces signes verbaux sont à prendre par le biais du signifiant, par le biais de leur matérialité plutôt que par celui de leur contenu, du signifié et du sens. Il s'apercevra d'ailleurs très vite qu'entre le signifiant S1 (qu'il s'agisse d'un signe corporel ou d'un représentant verbal peu importe) et le signifiant refoulé $S 2$ qui représente la trace verbale d'un souvenir pathogène, l'analyse ne pouvait se faire par une simple équation terme à terme: $\mathrm{S} 1$ = S2, par une simple traduction, voire une simple translittération, mais

10. Roman Jakobson (1963): «Deux aspects du langage et deux types d'aphasie», dans Essais de linguistique générale, Éditions de Minuit. 
qu'entre $\mathbf{S} 1$ et $\mathbf{S} 2$, il y avait toujours, en fait, une chaine plus ou moins longue, ordonnée (c'est-à-dire obéissant à une logique interne) de signifiants intermédiaires (Freud parle de représentations intermédiaires) :

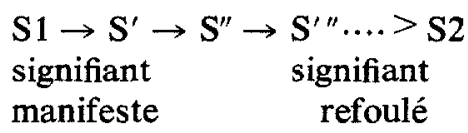

Il s'agit là d'indications fournies de la manière la plus claire dans le chapitre écrit en 1895 des Études sur l'hystérie et intitulé : Psychothérapie de l'hystérie. Tout lecteur tant soit peu sceptique pourra facilement s'y reporter et, de préférence à la traduction anglaise de Strachey, la traduction française étant à proprement parler innommable.

Ces signifiants intermédiaires - que Lacan nomme la chaîne signifiante - devront d'ailleurs être retrouvés dans l'analyse, indique Freud dans le même texte, suivant leur ordre propre, afin que le signifiant pathogène puisse être finalement retrouvé, voire reconstitué en vertu de l'ordonnancement logique qui préside à l'organisation de la chaîne.

Cette découverte que chaque analyste, à moins qu'il ne soit complètement sourd, refait quotidiennement dans sa pratique, des effets particuliers du langage et de sa traductibilité inhérente à sa structure (ce que Charles Sanders Peirce a infiniment mieux théorisé que Saussure grâce à son concept de sémiose), devait prendre une extension considérable lorsque Freud s'aperçut qu'il s'agissait là en fait des manifestations structurales du psychisme humain et qu'il n'est en fin de compte pas d'énoncé S1 proféré par le moi conscient d'un sujet - quel qu'il soit - qui ne renvoie en fait par le biais des chaînes signifiantes intermédiaires plus ou moins nombreuses et complexes, à un, ou des signifiants refoulés qui ne sont autres que les traces mnésiques de notre histoire passée, elles-mêmes ordonnées logiquement, quoique suivant une logique étrangère à celle de la conscience et de la ratio, en un système signifiant complexe : l'inconscient. Un inconscient, un «ça» devait-il le nommer plus tard d'après Groddeck, dont Freud devait découvrir le déterminisme radical sur l'ensemble de notre vie, en faisant l'analyse des rêves (l'Interprétation des rêves), des actes manqués, des lapsus, etc. (Psychopathologie de la vie quotidienne) et des mots d'esprit (le mot d'esprit dans son rapport avec l'inconscient); puis ensuite des productions culturelles en général (la Gradiva, Moïse et le monothéisme etc.) et... de la civilisation dans son ensemble (l'Avenir d'une illusion, Malaise dans la civilisation, Totem et tabou).

Mais il devait découvrir beaucoup plus et, en particulier, trois choses qui nous intéressent ici immédiatement :

1) que la partie consciente de la personnalité, le moi, qui produit les énoncés manifestes, doués d'un sens qui, comme Saussure l'a bien montré, est en grande partie fonction du contexte immédiat de l'énoncé (valeur du signe), et, comme on l'a montré depuis, du contexte socio-culturel de l'énonciation (la connotation dont nous reconnaissons volontiers avec Barthes la fonction essentiellement idéologique au point que l'analyse des idéologies passe par 
l'analyse des connotations) ${ }^{11}$, un sens multiple, donc, qui déborde considérablement le signifié; que ce moi est en fait définissable en termes de mécanismes de défense. Mécanismes dont on peut trouver la liste dans l'étude que leur a consacrée Anna Freud ${ }^{12}$ : refoulement, régression, formations réactionnelles, isolation, annulation rétroactive, projection, introjection, retournement sur soi, renversement dans le contraire, sublimation...; qui portent tous sur les chaînes signifiantes et ont pour fonction d'empêcher le retour dans le conscient des représentants-représentations (des signifiants) refoulés, autrement que sous une forme profondément remaniée. Leur fonction fait de ces défenses autant de manifestations langagières, de distorsions, de déplacements et de modifications des signifiants refoulés... disons qu'on y retrouverait sous des formes infiniment nuancées l'ensemble des fautes qu'un traducteur peut commettre, et saint Jérôme sait qu'elles sont nombreuses! dont on trouvera quelques exemples dans le texte ci-dessous de Patrick Mahony.

2) Que les signifiants refoulés sont des représentants de traces événementielles ou fantasmatiques liées à des affects, à des pulsions érotiques ou mortifères inacceptables par le moi conscient (c'est-à-dire le reflet sur le sujet des normes sociales) et ses super-structures répressives (moi idéal, idéal du moi et surmoi), et qui appartiennent, en général, aux tout débuts de notre histoire. Ces signifiants sont eux-mêmes organisés dans l'inconscient selon une logique propre, fait remarquer Freud dans son chapitre sur la Psychothérapie de l'hystérie, autre que celle qui préside à l'ordonnancement de nos représentations conscientes. C'est ce que Lacan a résumé dans une formule dont l'effet de scandale me déroutera toujours tant ce qu'elle résume est évident, littéral, chez Freud : «l'inconscient est structuré comme un langage». Ce que Lacan représente par un algorithme imité de celui de Saussure: $\frac{S}{S}$ dans lequel le $S$ représente la chaîne plus ou moins rationelle du discours moïque, $s$, l'ordonnancement logique de la chaîne signifiante refoulée et _-, la barre du refoulement.

3) Que pour que la barre du refoulement soit franchie, il faut que la continuité «logique » de la chaîne signifiante $S 1, S^{\prime}, S^{\prime \prime}, S^{\prime \prime}$ etc. qui mène à $S 2$, soit reconstituée. Il s'agit là, nous l'avons vu, de quelque chose de beaucoup plus complexe qu'une simple traduction, encore que l'ensemble des opérations de traduction puissent, à un titre ou un autre, y intervenir. Mais il faut qu'en outre quelque chose d'autre que ces simples jeux de langage quelle que soit leur ampleur soit pris en compte, quelque chose qui a effrayé Breuer et qui, étant donné la nature sexuelle et mortifère du matériau refoulé, puisque c'est précisément toujours de cette nature que s'avère le refoulé dans la pratique, se manifeste par l'établissement entre l'analyste et l'analysant en quête de ses signifiants refoulés, d'une relation dite transférentielle profondément marquée d'érotisme et de mort. Il s'agit du transfert psychanalytique, qui n'a absolument rien à voir avec les tendres sentiments à l'eau de rose qu'une mauvaise littérature pour mass media se plaît à décrire, et qui, tant du côté de l'analysant que de

11. Comme, par exemple, dans Mythologies, Seuil, 1957.

12. Anna Freud (1949): le Moi et les mécanismes de défense, Gallimard. 
celui de l'analyste est une véritable «mise en acte de l'inconscient» ${ }^{13}$, une activation des pulsions érotiques et mortifères qui mobilise les chaînes signifiantes refoulées.

Prendre le transfert en compte signifie qu'il sera d'abord reconnu puis que tout ce qui sera dit, de part et d'autre, ne prendra tout son sens et surtout sa portée et son efficacité que dans et par le transfert tant de l'analysant que de l'analyste. Le dire de l'analyste n'ayant d'autre fonction que de reconnaître, en la ponctuant, la reconstitution de la chaîne signifiante. Ce dire de l'analyste a une double fonction : 1) actualiser l'altérité radicale de toute parole et, 2) favoriser, par l'interprétation et surtout sa propre suspension (le silence de l'analyste) l'émergence de la chaîne signifiante : le passage de $S^{\prime}$ à $S^{\prime \prime}$, puis de $S^{\prime \prime}$ à $S^{\prime \prime \prime}$ et ainsi de suite jusqu'à ce que soient reconnus, démasqués, les $S 2$ refoulés constitutifs de l'inconscient. Les mécanismes de défense, les «fautes de traduction» et la logique qui préside à l'organisation de l'ensemble ayant été déconstruits, pièce par pièce.

On voit bien que la prise en considération du transfert fait de la relation analyste/analysant quelque chose de radicalement différent de toute autre relation à deux, qu'elle soit d'objectivation comme dans la relation classique médecin/malade ou imaginaire comme la relation amoureuse, où, dans tous les cas, le sujet de l'inconscient et les traces signifiantes dont il marque le discours moïque, défensif, conscient et plus ou moins rationnel, ne sont non seulement jamais pris en compte mais de surcroît toujours méconnus.

Il s'agit là d'une relation (la relation analytique) plus complexe où quatre pôles doivent être repérés si l'on veut apprécier les jeux complexes de la parole dans toute leur ampleur et repérer la position de qui parle dans le discours et de quelle place, puisque ce qu'en fin de compte Freud devait découvrir au-delà des principes économiques et dynamiques présidant aux destinées du sujet, est une véritable topologie du sujet, jusque-là inouie, et dont la découverte devait amener un profond remaniement des conceptions économiques et dynamiques.

Le schéma de Lacan nous est ici d'une aide précieuse ${ }^{14}$ :

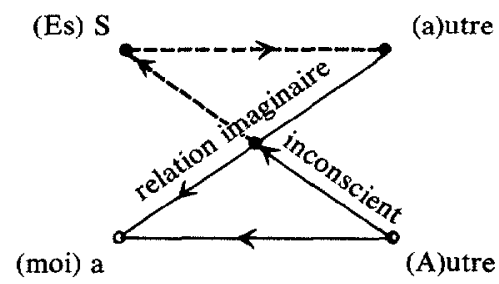

Je l'expliquerai par un exemple personnel : l'analyse sommaire d'un fantasme. Je me trouve en vacances pendant quelques jours en compagnie d'un autre (en haut et à droite sur le schéma), avec lequel j'entretiens des relations d'ami-

13. Définition que Lacan a donnée du transfert dans son séminaire sur le transfert 1960-61, non encore publié.

14. Le schéma $\mathbf{L}$, que l'on trouvera sous sa forme complète, ici reprise, à la page 53 des Écrits, Seuil, 1966. 
tié, d'ordre imaginaire, c'est-à-dire continuellement nourrie des images, des représentations de moi-même et de mon monde qui, pour être de l'ordre d'une méconnaissance des signifiants refoulés qui me déterminent $n$ 'en est pas moins tenace, puissante et investie de toutes sortes d'affects. Mon moi conscient est en bas, à gauche sur le schéma, la relation imaginaire d'amitié est tendue de l'autre à moi. Par imaginaire, je n'entends pas ce que ce mot désigne dans le langage courant. Je ne dis pas que mon amitié est imaginaire, elle est bel et bien réelle, mais s'organise autour de la catégorie de l'imaginaire qui est l'une des trois catégories qui, avec le symbolique et le Réel me structurent. C'est donc un ami que cet autre, presque un frère, une sorte d'alter ego qui m'est aussi familier, du moins $\mathrm{j}$ 'aime à le croire, que mon image dans le miroir. Dans son amitié pour moi, je me reconnais, je (re)trouve mon image.

Un soir, cet autre sort seul. Je ne l'accompagne pas. «Je rentrerai tard, me dit-il, ne t'occupe pas de moi». Le lendemain matin, je m'aperçois qu'il n'est pas rentré. Je pense d'abord qu'il a rencontré quelque bonne fortune et je m'en réjouis pour lui. Les heures passent et curieusement des images émergent et m'assaillent qui m'étonnent et que pourtant, d'une certaine façon, je reconnais parce qu'elles sont toujours là, dans mon imaginaire, prêtes à entrer en scène lorsque je suis en situation d'attendre quelqu'un, d'attendre l'autre. Ce ne sont pas tant des images d'ailleurs que des fragments de récits possibles, des bouts de phrases qui empruntent un contexte vraisemblable. Par exemple, dans le cas dont je parle, nous sommes à New York qui est une ville réputée dangereuse, encore que je n'y aie jamais fait l'expérience de cette dangerosité. L'axe de la relation imaginaire est, du fait de l'absence de l'autre, parcourue, tissée

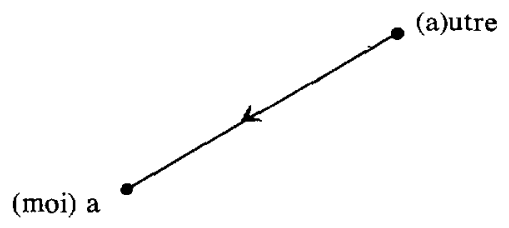

de ces fragments de propos:

"il y a eu une attaque..."

«il a été blessé, peut-être tué »

«le Roosevelt...»

«le téléphone sonne, une voix de femme, une infirmière, peut-être: on a trouvé votre nom sur le corps de... vous êtes bien Monsieur Peraldi... vous êtes prié de venir tout de suite...»

«Ce téléphone : j'ai le flash d'un ancien téléphone à manivelle..."

«New York Police... mais l'image qui me vient est celle d'un homme portant képi et vêtement bleu sombre qui n'est pas l'uniforme des policiers new yorkais...

«L'homme en uniforme a un air de circonstance... je devine la vérité de la mort de l'autre».

«Il a été fait prisonnier par des êtres à part, pas vraiment des criminels de la maffia, mais des fanatiques, cruels, inaccessibles et qui torturent $»$.

«Il faudra le venger, tous les tuer...»

«Il va falloir que je parte à sa recherche...» 
Tout ceci, bien sûr, en quelques minutes. Des flashs fulgurants auxquels, bien entendu, je ne crois pas. Je peux rationaliser ma crainte, elle est relativement fondée et une légère inquiétude, à New York, est, dans ce cas, de mise, par contre, rien ne peut vraiment justifier l'aspect déplacé, et dévastateur de ces pensées fugitives, chargées d'une étrange intensité, d'une densité qui tranche avec leur évanescence.

Sur ces entrefaites l'ami rentre. Ces images, instantanément s'évanouissent du moment que j'entends sa voix m'interpeller joyeusement pour me raconter sa nuit.

Des images de ce genre, des fantasmes qui nous viennent d'ailleurs à certains moments, à n'importe quel moment, en fait, de notre vie consciente lorsqu'il se produit un événement qui sort tant soit peu de notre routine, nous en possédons tous. Elles constituent la trame de notre imaginaire et ont toujours ce caractère profondément ennuyeux de la stéréotypie la plus banale. Tout imaginaire, contrairement à l'imagination, est relativement pauvre et essentiellement répétitif. Dans certains cas, dans l'hystérie par exemple, ou dans les névroses en général, ces fantasmes peuvent prendre une importance tout à fait dévastatrice et un caractère de réalité affective extrêmement douloureux. Même si le sujet qui s'en trouve envahi reconnaît bien là un phénomène excessif, il ne peut toutefois s'en défendre, et peut sentir croitre en lui une angoisse insurmontable. Ce n'était pas mon cas ce matin-là, encore qu'un certain potentiel d'angoisse vibrait derrière ces quelques fragments qui semblaient surgir de nulle part.

Ceci s'est produit pendant que je travaillais sur ce texte-ci comme pour me fournir un exemple de ce que je tentais de développer quant aux rapports de la parole et du sujet lorsque l'on prend en compte l'inconscient, un peu comme si l'inconscient sollicité par mon questionnement à son sujet, me faisait le don d'une ombre projetée d'un moment de mon ancienne névrose, celle-là même qui devait m'amener à devenir analyste. D'une certaine manière ces fantasmes m'ont intrigué et un peu amusé dans l'après-coup et $\mathrm{j}$ 'ai trouvé là l'occasion d'y revenir afin d'illustrer mon propos. Ces images, bien sûr, je les connais, elles m'accompagnent depuis longtemps et c'est en général l'absence et l'attente d'un «autre» masculin qui le déclenche. L'absence, le retard d'une femme, même aimée, ne déclenche rien de ce genre, c'est autre chose.

Il y a dans ces images quelque chose de violent, d'intense, d'archaïque de l'ordre du sentiment de fin du monde qui semble caractériser le chagrin des jeunes enfants. Cette réflexion fait resurgir l'image de l'homme en uniforme, je le reconnais, c'est un gendarme comme j'en ai connu en France, dans mon enfance. Si je précise ma vision, il pourrait s'agir du brigadier de gendarmerie de Saint-Rémy sur Avre, monsieur Salaud... "Quel nom, pour un brigadier de gendarmerie!» dit, dans mon souvenir, ma mère en riant.

Sur cette chaîne signifiante amorcée, la vérité soudain explose, le mur de l'imaginaire se déchire et, du champ de l'Autre (en bas à droite sur le schéma), de cet ailleurs radical de la parole, actualisé aujourd'hui dans ce voyage-ci par mon compagnon de voyage en son absence, des signifiants se détachent et se présentent autrement : je vais en donner quelques exemples, simplifiés : 
«Il y a eu une attaque...», c'est ce qu'on disait pendant la guerre (celle de 39-40), pendant laquelle d'ailleurs nous vivions à Saint-Rémy sur Avre alors qu'effectivement monsieur Salaud était brigadier de gendarmerie.

"Le Roosevelt...», c'est le Roosevelt Hospital où $j$ 'ai fait une conférence une fois à New York et où j'ai parlé d'un autre souvenir d'enfance qui concerne ma mère et mon rapport à la langue anglaise qu'elle parlait elle-même couramment lorsqu'elle était jeune. Hospital $\rightarrow$ hôpital, infirmière $\rightarrow$ infirmier. Lorraine.

Pendant la guerre, mon père était infirmier dans un hôpital, quelque part en

«Sans nouvelle, il va falloir que je parte à sa recherche...». C'était le téléphone chez mes grands-parents, qui tenaient un commerce, qui apportait des nouvelles pendant la guerre, un vieux téléphone à manivelle. C'était aussi le brigadier de gendarmerie, monsieur Salaud qui, s'il apportait des nouvelles, étaient alors de mauvaises nouvelles, c'est lui qui annonçait aux familles la mort des soldats. Sans nouvelle de mon père, ma mère était partie à sa recherche. Elle l'a retrouvé, en pleine guerre, a passé une nuit près de lui pour revenir immédiatement auprès de nous.

«Les êtres à part, fanatiques, cruels... torture», c'étaient les mots que l'on utilisait pour les SS, les nazis. "Tous les tuer!» paroles qui n'avaient pas grand sens pour moi qui les récoltaient à l'époque, sans doute celles de mon grand-père qui était dans la résistance.

En $S$, le (es), le ça, l'inconscient se structure autour de l'identification symbolique à ma mère. Dans mon attente de l'autre, je redeviens en fait ma propre mère dont je partageais l'attente anxieuse de mon père, en son absence, pendant la guerre, j'avais alors entre 17 et $\mathbf{2 6}$ mois. Attente angoissée, j'adorais mon père, redoublée de l'angoisse de ma mère et de ses signifiants à elle, car tous ces signifiants gravés en moi dès cette époque : hôpital, infirmier, guerre, nouvelles, nazis, etc., n'avaient pas de sens pour moi. Mais une angoisse dont le refoulement s'explique par son ambivalence. J'étais alors en plein odipe, seul avec ma mère, et certainement j'étais ravi de l'absence de ce père. Qu'il y reste à cette guerre! qu'il ne revienne pas! Tel était en réalité mon souhait comme en témoigne mon refus absolu de le reconnaître lorsqu'il est revenu de la guerre 9 mois après son départ. Les signifiants, gravés en moi dès cette époque, attendent en $\mathrm{A}$, au lieu de l'Autre, refoulés, le moment où, dans l'occasion transférentielle de mon rapport à un quelconque autre, qui me fut fournie ce matin-là par l'absence de l'ami, la situation d'attente les mobilisera ou plus exactement mobilisera la chaîne signifiante dans laquelle ils sont pris. C'est à moi qu'il appartiendra de saisir, ou non, cette chaîne par le bon bout, par le bout transférentiel si j'ose dire, ici la New York Police qui s'enchaîne à un autre signifiant, visuel, celui d'un homme en uniforme de gendarme, et de laisser cette faute logique (d'un point de vue référentiel strict) vibrer pour que, signifiant par signifiant, la chaîne se reconstitue suivant le parcours des traces mnésiques refoulées : les gendarmes de Saint-Rémy sur Avre, le brigadier monsieur Salaud, «quel nom pour un gendarme» (voix rieuse de ma mère), la guerre, les nouvelles de mort, etc. 
Nous pouvons situer le début de la châne sur notre schéma au point où l'axe de la relation imaginaire (qui comporte le signifiant New York Police), croise l'axe du discours de l'inconscient tendu de l'Autre au S. (et qui comporte le signifiant visuel d'un uniforme de gendarme, ici inapproprié au contexte newyorkais où mes fantasmes se déroulent, mais néanmoins lié, par association, au signifiant New York Police).

C'est ainsi qu'en psychanalyse, la parole fonctionne sous la forme $d$ ' «une interruption de la parole pleine entre le sujet de l'inconscient (S) et l'Autre», et de son détour par la relation imaginaire tendu du moi à l'autre, en l'occurrence l'ami, le compagnon de voyage auquel je suis lié par des sentiments d'affection.

Mais c'est ainsi qu'en fait toute parole fonctionne à ceci près qu'ici, $j$ 'ai considérablement simplifié le processus d'émergence de la chaîne signifiante refoulée et du souvenir afférent de l'attente de mon père, mais aussi de l'angoisse ambivalente puisque la tendre affection qu'elle semble indiquer recouvre en fait des souhaits de mort à son endroit qui furent, pendant très longtemps, complètement refoulés et parfaitement dévastateurs. S'ils sont ici presque à portée de la main, c'est que ce chemin ne m'est pas étranger mais j'ai encore vivace le souvenir des résistances qu'il m'a fallu franchir pendant le cours de mon analyse pour retrouver ce qui constituait le cœur de mes sentiments à l'endroit de mon père et la position dans laquelle ces sentiments m'avaient placé du fait des circonstances de son absence pendant la période où ils se constituaient et où je me constituais comme sujet. Ce qui dans tout cela fut sans doute le moins facile à assumer, fut précisément cette position féminine d'identification à ma mère, en $S$, car c'est là que, comme sujet de l'inconscient, je m'évanouis en quelque sorte, je m'efface pour n'être plus que le théâtre où se joue un drame qui n'est pas le mien, où se disent des mots qui ne m'appartiennent pas et dont je ne retiens que la forme signifiante et où je ne suis plus que l'écho de la détresse de ma mère, marqué de ces signifiants à elle certes, mais, du fait qu'ils appartiennent à la langue que je partage avec elle, toujours prêts à resurgir de l'altérité absolue qui est le lieu de dépôt des signifiants, ce lieu d'où semblent surgir les fantasmes comme il arrive quand on dit qu'un mot, une image, s'impose à notre esprit sans qu'on sache trop pourquoi ni d'où il vient. C'est précisément parce qu'il vient de l'Autre, le dépôt, le trésur des signifiants, ce lieu qui appartient à tout le monde et à personne et où, pour chacun d'entre nous, certains signifiants cristalliseront, hors du champ de notre conscience, tel ou tel moment crucial de notre histoire en ce qu'ils auront marqué les étapes de notre structuration comme sujet.

Si toute parole est ainsi perpétuellement en quête d'un dire qu'elle ne parvient jamais à formuler parce que plus ou moins refoulé, sauf en certains points de rencontre - comme celui de ces signifiants : New York Police $\rightarrow$ Uniforme de gendarme $\rightarrow$ Salaud, brigadier de gendarmerie $\rightarrow$ «voix rieuse de ma mère $\rightarrow$ guerre $\rightarrow$ Roosevelt $\rightarrow$ Roosevelt Hospital $\rightarrow$ Hôpital $\rightarrow$ infirmièr(e) $\rightarrow$ infirmier $\rightarrow$ père, etc., où s'opère une soudaine bifurcation dans le flot des pensées de la relation imaginaire et où s'amorce la chaîne signifiante qui se reconstitue chaînon par chaînon pour me mener vers ces pensées inconscientes 
qui me hantent et me déterminent dans les actes les plus menus de ma vie quotidienne, nous pouvons mieux dégager le double statut de la parole. D'une part cette parole vide de la relation imaginaire, de l'autre la parole pleine à laquelle le flot continu de la parole vide fait obstacle, sauf en certains points de croisement, où certains signifiants de la parole vide s'associent à un signifiant de la parole pleine, appelons-les, avec Lacan, les «points de capiton» ${ }^{15}$. Ce sera, dans mon exemple la collusion New York Police / Uniforme de gendarme.

On voit tout de suite le problème que ce statut de la parole peut soulever pour le traducteur, pour ne pas parler ici du linguiste, lui pour qui le signifiant est toujours ce qui se perd dans le transfert de sens qu'il opère d'une langue dans une autre, puisque ce qu'il perd est précisément ce autour de quoi se cristallisent les investissements pulsionnels inconscients qui nous déterminent, orientent sans cesse notre propos et témoignent indirectement de notre présence comme sujet sous les apparences trompeuses de notre moi.

$\mathrm{Si}$, par exemple, ce sont les voyelles claires ${ }^{16}$ qui constituent chez Verlaine ces points de capiton, si ce sont elles qui donnent à entendre sous le discours esthético-moïque de Verlaine, le discours infiniment plus mystérieux, mais aussi plus riche des investissements pulsionnels qui l'habitent, le discours du sujet de l'inconscient qui se profile en filigrane dans les textes poétiques, si ce sont elles - et nous pensons que ce sont effectivement elles - qui donnent au dire verlainien sa musique très particulière, sa vie, sa pulsation inconsciente (ce qu'on peut vérifier à tout moment et à n'importe quelle page en remarquant leur emploi presque excessif et les modulations extrêmement subtiles auxquelles elles sont soumises), alors le traducteur qui laisse tomber, au profit du sens à conserver, ces structures phonétiques si particulières, si propres à Verlaine, laisse tomber la parole pleine, la parole vraie, la parole vivante de Verlaine au profit de la parole vide, d'un monde de représentations imaginaires qui dans la langue d'arrivée - n'intéressent personne et ne relèvent en fin de compte que d'une idéologie aussi douteuse que surannée.

La conception psychanalytique de la parole, telle que je viens de l'esquisser, si elle doit être prise au sérieux par le traducteur, implique qu'à tout le moins, outre le signifié (auquel on le dresse à répondre par un autre signifiant), la valeur (pour laquelle on lui demande de lire plusieurs fois les textes avant de les traduire) et la connotation (pour laquelle il lui faut rétablir les «paramètres situationnels du message»), il se mette également à l'écoute du signifiant et que, d'une manière ou d'une autre, il réussisse à lui faire effectuer cette traversée qu'on nomme traduction.

D'une certaine manière on pourrait concevoir la parole comme une sorte de pièce de monnaie (on sait que la métaphore est de Saussure et qu'elle n'est pas sans intéresser aussi le psychanalyste) de taille variable. Le traducteur opérerait côté face, c'est-à-dire du côté de l'image, dans la dimension imaginaire du discours (c'est-à-dire l'ensemble des représentations plus ou moins idéologiques

15. La théorie du point de capiton est reprise et commentée par Lacan dans le séminaire sur Les psychoses, Seuil, 1981.

16. Voir, par exemple, les études de Jean Pierre Richard sur Verlaine. 
et plus ou moins scientifiques de son monde et de la place qu'il y occupe); alors que l'analyste opérerait côté pile, du côté du chiffre, du côté du signifiant qui, indépendamment du signifié dont il est toujours nécessairement orné, ne représente le Sujet (de l'inconscient) que pour un autre signifiant. Formule de Lacan que mon exemple illustre, je pense, assez clairement. Les signifiants de la chaîne représentant le sujet qui me structure mais qui est également le point où, identifié à ma mère dans l'attente angoissée de mon père, ce sujet en quelque sorte s'efface, s'évanouit devant d'autres signifiants, les siens à elle, de cette époque-là, ceux de son amour pour mon père et de son attente angoissée de ses nouvelles, de son retour : hôpital, infirmier, blessure, mort...

$\mathrm{Au}$ bord de la pièce, là où les deux faces se rejoignent, le traducteur et le psychanalyste partageraient ce qui pour l'un est faute de traduction et pour l'autre, l'un ou l'autre des mécanismes dits de défense. Si dans un texte sur la criminalité à New York, tel traducteur traduit «policeman»par «gendarme» il commet un faux-sens. Lorsque dans mon attente surgit au sein du flot de pensées plus ou moins angoissées le mot "New York Police" et que je luis associe l'image d'un homme en «uniforme de gendarme des années quarante», il y a déplacement et le signe qu'il $y$ a là l'amorce d'une chaîne signifiante possible.

Si un traducteur traduit "he passionately disliked his father» par «il aimait passionnément son père», il fait un contresens. Si dans mon accès transférentiel je semble retrouver dans mon attente angoissée l'image de mon père absent, le souvenir de mon amour pour lui et la crainte qu'il ne lui arrive quelque chose, il y a là un renversement dans son contraire car, au-delà de ce souvenir-écran, une fois d'autres résistances franchies, il apparaîtra tout au contraire que mon angoisse est liée à la crainte du châtiment qui double mes souhaits de mort à son endroit.

Je pourrais passer en revue toutes les fautes possibles de la traduction et montrer comment elles trouvent leur pendant dans l'un ou l'autre de ces mécanismes constitutifs du refoulement. Peut-être, précisément, parce que lorsque son attention au sens fléchit, le traducteur rejoint, sans même s'en apercevoir, la position du psychanalyste et qu'il se met alors à l'écoute de quelque chose d'autre que ce qu'il a, effectivement, à traduire. Quelque chose d'autre mais qui cependant est également là dans le texte. Toutefois, à la différence du psychanalyste, il ignore, s'il n'est pas analysé, quels sont les signifiants qui lui sont propres et qui, dans ces mouvements de relâchement de son attention ou, à l'inverse, de brusque tension transférentielle à l'endroit du texte qu'il traduit (je pense à l'intensité du transfert de Gérard de Nerval traduisant le Faust de Goethe), vont se précipiter soudain, à l'appel de certains signifiants du texte qu'il traduit, sous sa plume et se retrouver, inopportuns et incongrus, dans le texte d'arrivée.

Dirons-nous, non sans quelque fatalisme, que l'on ne peut pas tout faire, que la traduction, comme chacun le sait, est trahison, et refermer le livre comme le poète, sur le nom de Paphos? Ce serait fermer bien rapidement les yeux sur le fait que ce qui se perd, ce faisant, est la vie même du texte, sa vérité la plus essentielle, sa plénitude, ce serait toujours favoriser le discours 
de l'idéologie contre celui du désir. Quelqu'un comme Ezra Pound, qui n'était pas psychanalyste, mais assurément le plus grand poète américain du vingtième siècle et, de surcroît, un traducteur hors pair, avait de la langue un amour beaucoup trop exigeant pour fermer les yeux sur cette question.

L'une des questions que se posait Pound, par exemple, fut de savoir pourquoi Dante avait parlé de certains poètes et, notamment, des troubadours. Poètes bien particuliers dans ce sens qu'ils sont les seuls écrivains (en tout cas jusqu'à Bataille, et encore!) qui, comme les psychanalystes, mais sept siècles auparavant, ont tenté d'élaborer une «érotique», c'est-à-dire de construire des textes qui ne parlent pas de l'amour, mais qui sont paroles d'amour, paroles amoureuses. Leur propos était de produire un discours entièrement libidinalisé. Or, ils ont très bien compris qu'un discours n'est pas libidinalisé, érotisé, du fait des représentations qu'il propose, au niveau du signifié, au niveau du sens. Rien de moins pornographique que la poésie des troubadours et pourtant rien de plus érotique. C'est au niveau de la structure signifiante qu'ils ont accompli leur travail. «Il semble, remarque Pound, que toute la culture de l'époque, ou du moins la grande masse de la culture purement littéraire de l'époque, à partir de 1080 jusqu'en 1250 ou en 1300 , se concentrait sur un seul problème esthétique qui, pour reprendre l'expression de Dante, concernait l'art tout entier. Cet art fut celui de mettre ensemble à peu près 6 strophes de poésie en faisant fusionner paroles et musique de telle sorte que ni défaut ni soudure ne se visse». Par musique, Pound entendait très exactement ce qui structure, ce qui scande le signifiant pour lui donner sa valeur et sa portée propre, qu'il s'agisse du rythme ou de la tonalité des phonèmes. «Qu'est-ce qu'ils savent de l'amour et qu'est-ce qu'ils peuvent en comprendre, demande Pound dans Mauberley, s'ils ne comprennent pas la poésie, s'ils ne sentent pas la musique, qu'est-ce qu'ils peuvent comprendre de cette passion en comparaison avec laquelle la rose est grossière et le parfum des violettes un tonnerre? » Intuition profondément psychanalytique des fonctions véritables de la parole et du niveau où toute parole devrait être entendue et surtout la parole d'amour qui, contrairement à l'opinion la plus répandue, n'est en aucune façon cette sorte d'épanchement nauséabond et informe auquel les mass media nous exposent, mais est rigoureusement structurée, de la façon la plus exigeante (celle-là même où seuls les troubadours ont excellé), selon une véritable logique du signifiant: c'est cela une érotique, à savoir la tentative de dégager la logique des investissements pulsionnels du signifiant et de leur ordonnancement dans le progrès syntagmatique. C'est cela la contrainte rigoureuse à laquelle les troubadours se sont soumis et non, comme le veut l'imagerie populaire que nous en a transmis la caricature, quelque ascétisme charnel, quelque chasteté dont la ceinture des dames serait le pendant vestimentaire féminin.

Sans doute la chasteté attribuée faussement au troubadour connote-t-elle que pour lui ce n'est pas le signifié, le sens ou le référent (comme chez Sade) qui est érotique ou érotisable, mais le signifiant pris dans le mouvement du syntagme (ce mouvement que Pound nomme musique) : d'où l'importance de la césure, de la longueur des mètres, de la mise en opposition de certains groupements phoniques, des répétitions de certains sons, de la dominante momentanée, sur toute une strophe, de tel phonème ou de tel groupe de phonè- 
mes; et surtout l'importance de la logique qui préside à ces ordonnancements et dont seul le dégagement permet, si l'on désire véritablement traduire ces poèmes, la reconstruction d'un analogon dans la langue d'arrivée. C'est ce sur quoi, précisément, Pound a travaillé avec acharnement, lorsqu'il entreprit de traduire, après les avoir lus et critiqués. Lire c'est ici dégager, repérer la ou les structure(s) logique(s) prévalentes dans l'ordonnancement syntagmatique d'un texte, tant en ce qui regarde l'ordonnancement des signifiés (une logique sémantique) qu'en ce qui concerne l'ordonnancement des signifiants (une logique érotique). Quant à la critique chez Pound, pourquoi ne pas le signaler en passant, on sait qu'elle fonctionne à cinq niveaux :

1. Criticism by discussion, extending from mere yatter, logic-chopping, and description of tendencies up to the clearly defined record of procedures and an attempt to formulate more or less general principles...

2. Criticism by translation.

3. Criticism by exercise in the style of a given period...

4. Criticism via music, meaning definitely the setting of a poet's words... this is the most intensive form of criticism save :

5. Criticism in new composition... ${ }^{17}$

La traduction qu'il nous fournit de tel poème d'Arnaut Daniel, analysée musicalement par Stephen G. Nichols ${ }^{18}$, est un exemple intéressant de travail sur la traduction du signifiant :
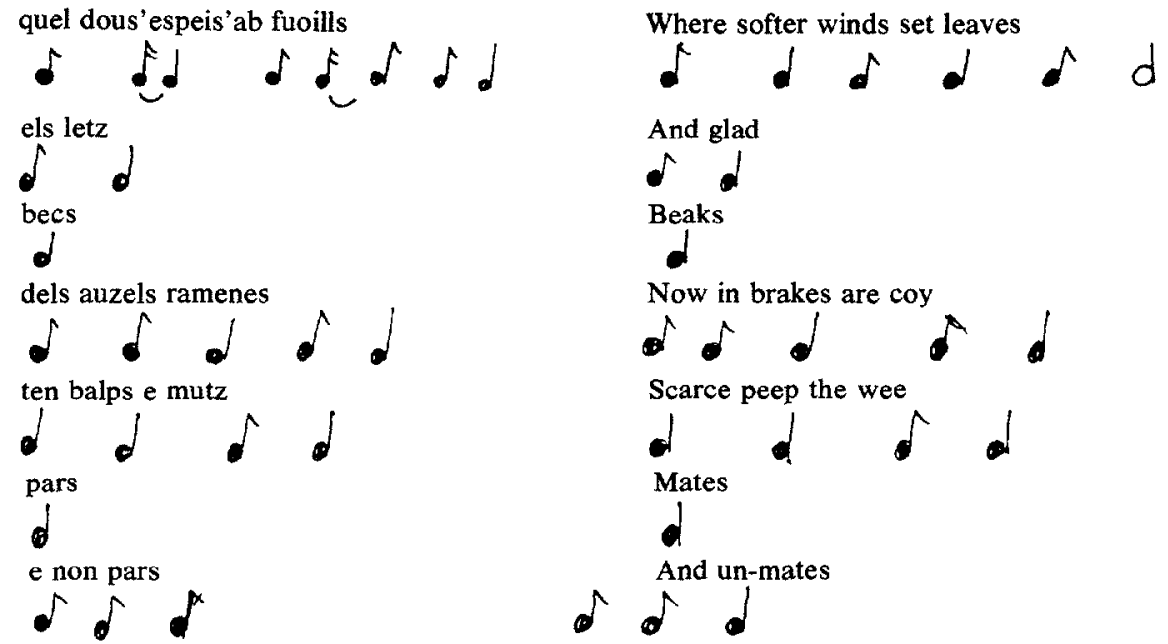

La poésie, comme toujours, est le lieu du texte où se manifeste de la façon la plus exemplaire la rencontre de l'intuition fulgurante du maître des mots et de l'érotique du signifiant avec la découverte patiente et précautionneuse, mais non sans passion elle aussi, par le psychanalyste, des lois du signifiant qui président à l'ordonnancement d'un inconscient, structuré comme un langage, certes, mais aussi où s'enchaînent et se déchaînent (syntagma en grec veut dire châ̂ne) les pulsions, fussent-elles érotiques et/ou de mort, qui somme toute, nous animent comme sujet.

17. Ezra Pound (1968) : Literary Essays, N.D.P.

18. Stephen Nichols (1965): «Lo Pounds si fol del Amerique», dans Cahiers de l'Herne $\mathrm{n}^{\circ} 7$, L'Herne. 